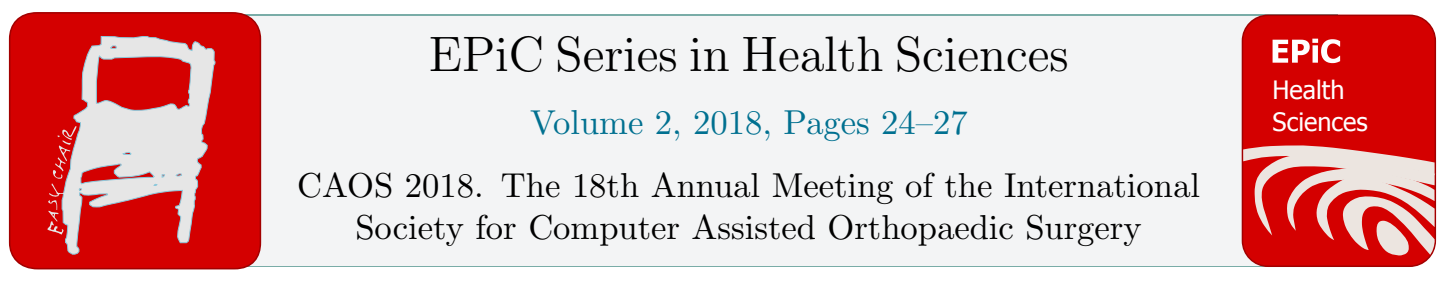

\title{
“Standard” Versus “Challenging” Patients - Perioperative Outcomes using Computer- Assisted Total Knee Arthroplasty
}

\author{
Yifei Dai ${ }^{1 *}$, Quanjun Cui ${ }^{2}$, Michael P Bolognesi ${ }^{3}$, Samuel S Wellman ${ }^{3}$, \\ Thorsten Seyler ${ }^{3}$, Yassaman Najmabadi ${ }^{1}$, Charlotte Bolch ${ }^{1}$, David Liu ${ }^{4 *}$ \\ ${ }^{1 *}$ Exactech Inc, Gainesville, FL 32653, USA \\ ${ }^{2}$ University of Virginia School of Medicine, Charlottesville, VA, USA \\ ${ }^{3}$ Duke Orthopaedics, Durham, NC, USA \\ ${ }^{4}$ The Gold Coast Centre for Bone and Joint Surgery, Queensland, Tugun, AU \\ yifei.dai@exac.com, dliu01@bigpond.com
}

\begin{abstract}
This study investigated if CAOS TKA cases in higher risk patients would impact the perioperative outcomes of surgery. Intraoperative and recovery/discharge data on 70 patients (72 knees) from a multicenter, consecutive series were analyzed. The patients were grouped into challenging and standard case groups according to the criteria of age, BMI, and degree of deformity. Despite a general trend observed between the challenging and standard cases, the two groups did not exhibit significant differences in terms of surgical time, blood loss, recovery and time to discharge. The data demonstrated consistent perioperative results by CAOS TKA irrespective of patient conditions.
\end{abstract}

\section{Introduction}

Total knee arthroplasty (TKA) performed with computer-assisted orthopedic system (CAOS) has been proven to significantly improve implant alignment consistency compared to conventional instrumentation [1,2]. Additionally, the benefits of CAOS TKA in the patients with extra-articular deformity has been published. However, most of these studies are case reports and single arm series focusing on alignment and short-term outcomes [3-5].

Results of conventional instrumented TKA concluded factors such as advanced age, obesity, and coronal deformity increased the risk of longer surgical times and length of stay, poor function and early failure [6-9]. These findings indicated that patient type or local condition of the knee may sometimes require special perioperative attention, which makes a challenging patient. To date, it remains unclear how perioperative outcomes are impacted by the challenging cases, as compared to the "standard" cases 
for CAOS TKA. This study analyzed a case series to investigate if there is a difference between challenging and standard cases in terms of perioperative outcomes, specifically in surgical time, blood loss, hospital recovery and discharge.

\section{Materials and Methods}

With institutional review board-approval and patient's signed informed consent, a multicentre, consecutive case series study was conducted by 5 surgeons in a total of 70 patients ( 72 knees). All cases were primary TKA using the Optetrak Logic Knee System (Exactech Inc, Gainesville, FL, USA) with the assistance of a contemporary CAOS system (ExactechGPS, Blue-Ortho, Gieres, FR). The two bilateral patients in this series had their knees operated separately with at least 90 days apart.

"Challenging" cases were defined from the series as having one or more of the following conditions: 1) age greater than 80 years, 2) BMI greater than 35,3 ) coronal deformity greater than $15^{\circ}$, and 4) range of flexion (ROM) less than $90^{\circ}$. Perioperative outcomes were compared between the standard and challenging case groups. Specific outcomes assessed were: surgical time, intraoperative blood loss, haemoglobin change (preoperative - postoperative), days to initial ambulation, hospital length of stay, and discharge destination. Statistical significance was defined as $\mathrm{p}<0.05$.

\section{Results}

Twenty-six knees were identified as challenging cases, whereas the remaining forty-six were grouped as standard cases (Table 1). The challenging case group consisted of more female patients, had a higher BMI, and more comorbidities compared to the standard group. A summary of perioperative outcomes in the standard and challenging groups is presented in Table 2. The challenging cases tended to have more intraoperative blood loss (by $24 \mathrm{ml}$ ) and a lower discharge to home rate (by 9\%) than the standard cases. Compared to the standard cases, a higher variability (standard deviation) was associated with the challenging cases in both intraoperative blood loss and haemoglobin change. The challenging cases tended to require a slightly longer surgical time (by $7 \mathrm{~min}$ ) than the standard cases. Despite the general trends found between challenging and standard cases, none of the differences were statistically significant. No blood transfusion or implant related early complications were reported in either group by the time of discharge.

\section{Discussion}

With a previous study proving consistent accuracy using CAOS in TKA performed by surgeons of varying experience levels [10], this investigation sought to investigate the sensitivity of the perioperative outcomes of CAOS TKA to patient conditions. Although the conditions used to determine challenging cases in this study (age, BMI, deformity) have been shown by previous clinical investigations to impact conventional TKA results [6-9], the present data did not suggest inferior perioperative outcomes in the challenging cases compared to the standard cases using CAOS. This may be due to the benefits of CAOS facilitating intraoperative surgical guidance to help the surgeon mitigate surgical challenges and uncertainties while operating on the challenging patients, as well as avoiding intramedullary instrumentation. Like previous case studies and clinical series that have proposed the benefits of CAOS in TKA cases with severe coronal deformity [3-5], this study highlights the advantages CAOS TKA may offer in demanding cases where patient or joint factors increase the surgical challenges. 


\begin{tabular}{lccc} 
A & & & \\
\hline Challenging Patients & Standard Patients & $P$ \\
Age (years, mean, range) & 26 & 46 & - \\
Female (\%) & $63.9 \pm 10.1,41-82$ & $67.5 \pm 8.5,51-80$ & 0.174 \\
BMI (mean, range) & 62 & 41 & 0.000 \\
Count of Comorbidities (mean, range)* & $35.7 \pm 6.2,26.2-47.0$ & $29.8 \pm 2.7,23.5-34.9$ & 0.005 \\
& $1.7 \pm 1.0,0.0-4.0$ & $1.1 \pm 1.0,0.0-3.0$ & 0.021 \\
\hline
\end{tabular}

\begin{tabular}{lc}
${ }^{*}$ The total number of comobidities per $\mathrm{p}$ \\
$\mathrm{B}$ \\
\hline \multicolumn{2}{c}{ Challenging Patients } \\
$\mathrm{N} \dagger$ \\
$\mathrm{Age}>80 \mathrm{yr}$ & 3 \\
$\mathrm{BMI}>35$ & 17 \\
Coronal Deformity $>15^{\circ}$ & 1 \\
$\mathrm{ROM}<90^{\circ}$ & 10 \\
\hline
\end{tabular}

† Each difficult case had one or more listed conditions.

Table 1. A) Patient conditions. B) Distribution of conditions in the challenging TKA group.

\begin{tabular}{lccc}
\hline & Challenging Pateints & Standard Patients & $\mathrm{P}$ \\
Intraop Blood Loss (ml) & $154 \pm 162$ & $130 \pm 139$ & 0.529 \\
Haemoglobin Loss (g/dL) & $2.1 \pm 2.9$ & $2.3 \pm 1.8$ & 0.700 \\
First Ambulation (day) & $0.4 \pm 0.5$ & $0.4 \pm 0.5$ & 0.914 \\
Length of Stay (day) & $3.3 \pm 1.5$ & $3.2 \pm 1.7$ & 0.915 \\
Discharge to Home (\%) & 69 & 78 & 0.149 \\
\hline
\end{tabular}

Table 2. Summary of perioperative outcomes for the challenging and standard TKA groups.

\section{References}

[1] Brin YS, et al. Imageless computer assisted versus conventional total knee replacement. A Bayesian meta-analysis of 23 comparative studies. Int Orthop,35(3):331-9,2011.

[2] Hetaimish BM, et al. Meta-analysis of navigation vs conventional total knee arthroplasty. J Arthroplasty,27(6):1177-82,2012.

[3] Mullaji A, Shetty GM. Computer-assisted total knee arthroplasty for arthritis with extra-articular deformity. J Arthroplasty. 2009;24(8):1164-9.

[4] Bottros J, Klika AK, Lee HH, Polousky J, Barsoum WK. The use of navigation in total knee arthroplasty for patients with extra-articular deformity. J Arthroplasty. 2008;23(1):74-8.

[5] Kuo CC, Bosque J, Meehan JP, Jamali AA. Computer-assisted navigation of total knee arthroplasty for osteoarthritis in a patient with severe posttraumatic femoral deformity. J Arthroplasty. 2011;26(6):976.e17-20.

[6] Teeney SM, Krackow KA, Hungerford DS, Jones M. Primary total knee arthroplasty in patients with severe varus deformity. Clin Orthop Relat Res 1991;273:19-31.

[7] Krushell RJ, Fingeroth RJ. Primary total knee arthroplasty in morbidly obese patients: a 5 to 14 year follow up study. J Arthroplast 2007;22(6 suppl 2):77-80. 
[8] Foran JR, Mont MA, Etienne G, Jones LC, Hungerford DS. The outcome of total knee arthroplasty in obese patients. J Bone Jt Surg [Am] 2004;86-A(8):1609-15.

[9] Smith ID, Elton R, Ballantyne JA, Brenkel IJ. Pre-operative predictors of the length of hospital stay in total knee replacement. J Bone Joint Surg Br. 2008;90(11):1435-40.

[10] Dai Y, Jung A, Hamad C, et al. Does Learning Curve Affect the Accuracy in Resection Alignment During Navigated Total Knee Artroplasty? $17^{\text {th }}$ Annual Meeting of the International Socienty of Compter Assited Orthopaedic Surgery. June 14-17, 2017. Aachen, DE. 